\title{
CAPITAL SOCIAL ORGANIZACIONAL EM UM PROJETO INTERORGANIZACIONAL: UM ESTUDO DESENVOLVIDO NO TERCEIRO SETOR
}

\author{
ORGANIZATIONAL SOCIAL CAPITAL IN \\ INTERORGANIZATIONAL PROJECT: \\ A STUDY DEVELOPED IN THE THIRD SECTOR
}

Data de submissão: 08-10-2014 Aceite: 21-07-2015

Rosana da Rosa Portella Tondolo ${ }^{1}$ Claudia Cristina Bitencourt ${ }^{2}$ Guilherme Luís Roehe Vaccaro ${ }^{3}$

\section{RESUMO}

As organizações do Terceiro Setor surgiram com o propósito de encontrar solução para os diferentes tipos de problemas sociais. Estas organizações têm como desafio manter suas atividades e atingir a sustentabilidade organizacional exclusivamente por meio da captação de recursos de terceiros, sendo estes originados a partir de doações, parcerias, convênios, entre outros. Nesse sentido, este estudo visou identificar o nível de Capital Social Organizacional (CSO) das organizações sociais participantes de um projeto interorganizacional, desenvolvido no Estado do Rio Grande do Sul. Para tal, foi realizada uma pesquisa quantitativa com 44 organizações, representando aproximadamente $58 \%$ da população. Os dados foram analisados por meio da análise de conglomerados e testes não paramétricos de Kruskal-Wallis e Wilcoxon -Mann-Whitney. As principais contribuições deste estudo envolvem a classificação de 3 grupos com níveis distintos de CSO, e a reflexão sobre as motivações e razões da existência desses grupos. Esta pesquisa apresentou o viés do respondente como um limitador. Ao final, são apresentadas as sugestões de estudos futuros, as quais envolvem a investigação das variáveis "visão compartilhada 2" e "confiança 2", e a relação entre os níveis de CSO e a forma como os recursos são mobilizados pelas organizações.

Palavras-Chave: Capital Social Organizacional. Organizações Sociais. Terceiro Setor.

\footnotetext{
1 Possui graduação em Ciências Contábeis pela Universidade de Caxias do Sul, UCS, mestrado em Administração pela Universidade de Caxias do Sul, UCS e doutorado em Administração pela Universidade do Vale do Rio dos Sinos, UNISINOS. Pelotas. Rio Grande do Sul. Brasil. E-mail: rosanatondolo@gmail.com

2 Possui graduação em Administração de Empresas pela Pontifícia Universidade Católica do Rio Grande do Sul, PUCRS, mestrado em Administração pela Universidade Federal do Rio Grande do Sul, UFRGS, doutorado em Business pela The University of Queensland, UQ, Austrália com período sanduíche em The University of Queensland e Doutorado em Administração pela Universidade Federal do Rio Grande do Sul, UFRGS. São Leopoldo. Rio Grande do Sul. Brasil. E-mail: claucbitencourt@gmail.com

3 Possui graduação em Bacharelado em Matemática Aplicada e Computacional pela Universidade Federal do Rio Grande do Sul, UFRGS, graduação interrompida em 1995 em Bacharelado em Estatística pela Universidade Federal do Rio Grande do Sul, UFRGS, mestrado em Engenharia de Produção pela Universidade Federal do Rio Grande do Sul, UFRGS e doutorado em Computação pela Universidade Federal do Rio Grande do Sul, UFRGS. São Leopoldo. Rio Grande do Sul. Brasil. E-mail: guilhermevaccaro@gmail.com
} 


\begin{abstract}
Third Sector Organizations have emerged with the purpose of finding solution to different kinds of social problems. These organizations are challenged to keep their activities and achieve organizational sustainability basically gathering founds from third parties, which are derived from donations, partnerships, agreements, among others. In this sense, this study aimed to identify the level of Organizational Social Capital (OSC) of social organizations that participated of an interorganizational project developed in the Rio Grande do Sul State, Brazil. In this regard, it was conducted a quantitative survey using cluster analysis and non-parametric tests such as Kruskal-Wallis and Wilcoxon-Mann-Whitney. The main contributions of this study are the classification of 3 groups with different levels of CSO, and the reflection on the motivations and reasons for the existence of these three different groups regarding level of CSO. This study showed the respondent bias as a limitation. Finally, it is suggested directions for future studies, which consider the examination of the variables shared vision 2 and trust 2, and the relationship between the levels of CSO and how the resources are mobilized by organizations.
\end{abstract}

Keywords: Organizational Social Capital. NonProfit Organizations. Third Sector.

\title{
1 INTRODUÇÃO
}

O conceito de capital social refere-se aos recursos reais e potenciais disponíveis e derivados da rede de relacionamentos, formadas pelo indivíduo ou unidade social (NAHAPIET; GHOSHAL, 1998). O capital social migrou para diferentes campos de estudo, ocorrendo, assim, uma adaptação de seu conceito e, mais do que isso, a criação de conceitos associativos ou até mesmo derivativos. Nesse ínterim, surgiu o conceito de Capital Social Organizacional (CSO), o qual relaciona o conceito de capital social ao contexto organizacional. Desta forma, os autores preconizadores do CSO, Nahapiet e Ghoshal (1998) abordaram o escopo estratégico a partir de premissas da Visão Baseada em Recursos (VBR).

O CSO tem sido rotineiramente abordado pela literatura à luz da VBR, por isso é possível identificar autores que apresentam as características da VBR para explicar as vantagens do CSO (ARREGLE et al., 2007; EDELMAN et al., 2004; LEANA; VAN BUREN, 1999; PENNINGS; LEE, 1998). O CSO consiste na boa vontade e nos recursos que as organizações acessam por meio de suas relações com outras organizações (ZAHRA, 2010). Nesse sentido, o CSO é visto como um facilitador, pois pode promover o acesso ao recurso externo, bem como facilitar processos organizacionais internos (ARREGLE et al., 2007; SIRMON; HITT; IRELAND, 2007). Dessa forma, o capital social, sob a ótica organizacional, tem sido ligado a fatores intra e extraorganizacionais, facilitando a formação, expansão, contração e gestão de organizações públicas, privadas e sem fins lucrativos (SAXTON; BENSON, 2005).

No Terceiro Setor, o CSO pode ser visto como um propulsor na busca pelo recurso, o que caracteriza um importante aspecto, uma vez que as organizações do Terceiro Setor apresentam recursos, na maioria das vezes, escassos, e demandas infinitas. Além disso, grande parte das organizações conta com o apoio de voluntários para o desenvolvimento de suas atividades (SALAMON, 2010).

Esse setor começou a se desenvolver a partir da necessidade social, uma vez que o setor público não atendia as necessidades da população. Assim, organizações ligadas ao Terceiro Setor surgiram com o propósito de buscar alternativas aos mais variados problemas sociais que envolviam as comunidades (SARAIVA, 2006). Em particular, com a finalidade de suprir um serviço público, as organizações da sociedade civil (OSCs) ocuparam um espaço que era de direito do Estado, sendo permeado por estas com o passar dos anos.

A fim de atingir seus objetivos sociais, as organizações do Terceiro Setor atuam em uma lógica colaborativa, desenvolvendo parcerias, convênios e termos de cooperação com organizações e instituições de diferentes esferas. Essas ações são realizadas visando à arrecadação de fun- 
dos para financiamento das operações desenvolvidas pelas organizações e, consequentemente, o atendimento de seu público-alvo.

Nesse sentido, as relações que essas organizações possuem podem ser consideradas como diferencial no momento da captação dos recursos, uma vez que as diversas interações realizadas com pessoas físicas, jurídicas e com o poder público são consideradas a origem no processo de captação de recursos. Estes recursos, capitaneados pela organização social, são revertidos em ações da organização e direcionados ao atendimento de seu público beneficiário e cumprimento de seu objetivo social (CRUZ et al., 2010).

Nessa lógica, as empresas com finalidade lucrativa estão buscando desenvolver capacidades para atender as demandas sociais e ambientais, uma vez que os consumidores e a comunidade do entorno realizam pressões quanto à responsabilidade socioambiental corporativa. Essa nova ótica privilegia os aspectos de captação de recursos para as organizações do Terceiro Setor, visto que muitas empresas preferem atender a essa demanda contribuindo para as organizações sociais por meio de parcerias e desenvolvimento de projetos (ORO, 2014).

Diante disso, este estudo apresenta a seguinte questão norteadora de pesquisa: Qual o nível de capital social organizacional (CSO) das organizações sociais participantes de um projeto interorganizacional desenvolvido no Estado do Rio Grande do Sul.

Em decorrência desse problema, este estudo objetiva identificar o nível de capital social organizacional (CSO) das organizações sociais participantes de um projeto interorganizacional desenvolvido no Estado do Rio Grande do Sul. Como objetivos específicos, busca-se (i) medir a média de CSO de cada organização; (ii) agrupar as organizações quanto aos seus níveis de CSO; e, (iii) analisar e discutir os resultados por dimensão do CSO (estrutural, cognitiva, relacional e mobilizadora).

Considerado o contexto previamente apresentado, o estudo do CSO é relevante, uma vez que o CSO pode ser considerado um propulsor, tanto na captação de recursos, quanto no desenvolvimento de projetos. Além disso, este estudo se justifica à medida que contribui para o avanço dos estudos no campo da gestão das organizações sociais, buscando identificar e avançar em algumas reflexões sobre lacunas até então ainda pouco exploradas pela literatura. Ademais, este estudo apresenta alguns direcionamentos para pesquisas futuras.

\section{CAPITAL SOCIAL ORGANIZACIONAL NO TERCEIRO SETOR}

O conceito de capital social organizacional (CSO) foi pioneiramente abordado por Nahapiet e Ghoshal (1998). Os autores apresentam um dos primeiros estudos relacionando o capital social ao capital intelectual na busca de vantagem organizacional. Os autores são considerados percursores na aplicação do Capital Social em Organizações, uma vez que apresentam o ambiente competitivo relacionando-o ao capital social das organizações. Ao definir o campo estratégico como escopo, os autores utilizam as premissas da Visão Baseada em Recursos (VBR), associando-as ao conceito de capital social. Dessa forma, percebe-se que o CSO tem sido rotineiramente abordado pela literatura à luz da VBR, no intuito de explicar as vantagens do CSO (ARREGLE et al., 2007; EDELMAN et al., 2004; LEANA; VAN BUREN, 1999; PENNINGS; LEE, 1998).

A maior parte dos estudos sobre CSO tem como escopo as organizações com fins lucrativos. Embora a VBR apresente preceitos estratégicos em ambientes competitivos (OLIVEIRA, 2008), acredita-se que algumas de suas premissas, principalmente no que tange o papel dos recursos e das capacidades, podem ser consideradas quando analisadas as organizações do Ter- 
ceiro Setor, ainda que ambientes competitivos não sejam o foco deste estudo, uma vez que as organizações sociais estão imersas em um ambiente colaborativo.

Com relação às Organizações do Terceiro Setor, é importante realizar duas reflexões. A primeira concentra-se na lógica colaborativa e cooperativa por elas apresentada, uma vez que suas ações, na maioria das vezes, visam o bem-estar da comunidade. Assim, perseguem objetivos coletivos, ou ainda, objetivos individuais, os quais podem ser comuns a outras organizações.

A segunda reflexão está nas especificidades das organizações do Terceiro Setor, uma vez que essas organizações apresentam características particulares, tais como: não visam lucro, atuam em benefício do outro, desempenham atividades de interesse público, parte significativa da renda é oriunda de doações, e a maioria de sua força de trabalho é voluntária. Além disso, o Terceiro Setor tem crescido nas últimas décadas e atualmente ocupa uma fatia considerável da economia, gerando emprego e renda, além dos benefícios sociais ocasionados pela sua atuação (SALAMON, 1994, 2010).

Devido à contribuição desse setor para a sociedade e sua participação na economia atual, é importante entender não somente a sua lógica, mas como o capital social, sendo considerado recurso, pode estar relacionado às organizações do Terceiro Setor. Nesse sentido, o CSO consiste nos recursos que as organizações desenvolvem por meio de suas relações com outras organizações (ZAHRA, 2010), ou ainda, pode ser interpretado como a boa vontade de disponibilizar recursos a um ator por meio de suas recíprocas relações de confiança (ARREGLE et al., 2007).

Buscando um melhor entendimento dos recursos nesse cenário, o CSO pode prover diferentes tipos de recursos para as organizações, tais como informações, conhecimento, tecnologia, capital financeiro, posicionamento em redes e relações com atores estratégicos (ARREGLE et al., 2007). O CSO pode, também, afetar positivamente as atividades internas e externas da organização (ARREGLE et al., 2007). Nesse sentido, o CSO pode ser visto como um recurso/capacidade, sob o ponto de vista da estratégia.

Neste contexto, a utilização da VBR como lente para entender o CSO pode ser aplicada também às organizações do Terceiro Setor, uma vez que essas organizações necessitam de recursos tanto quanto as organizações com fins lucrativos. As organizações do Terceiro Setor necessitam de recursos e capacidades para cumprir com sua função social e desempenhar seus objetivos organizacionais. Desse modo, os recursos e as capacidades internas da organização contribuem de forma efetiva para as estratégias e sustentabilidade das organizações sociais.

Sugere-se que o capital social é um recurso valioso porque pode reduzir os custos de transação, por meio da economia nos custos de informação e transação (NAHAPIET; GHOSHAL, 1998). De acordo com Nahapiet e Ghoshal (1998), as diferenças de desempenho encontradas entre as firmas podem ser explicadas pelas diferenças nas habilidades para criar e explorar o capital social. Nesse sentido, o CSO é um importante recurso intangível, que pode levar anos para ser desenvolvido e que pode ser potencializado, tornando, assim, seu valor mais duradouro, devido a sua dependência de trajetória organizacional (ZAHRA, 2010).

É possível identificar, na literatura, estudos que compreendem o capital social e/ou o CSO como recurso, sendo que este contribui de forma significativa para a vantagem organizacional (ADLER; KWON, 2002; ARREGLE et al., 2007; BOLINO; TURNLEY; BLOODGOOD, 2002; LEANA; VAN BUREN, 1999; NAHAPIET; GHOSHAL, 1998; TSAI; GHOSHAL, 1998). No entanto, refletindo sobre a ótica do contexto social, Saxton e Benson (2005, p. 19) defendem que "o capital social constitui um recurso considerável para o desenvolvimento social, político e econômico de uma comunidade". Diante disso, o capital social liga-se a fatores internos e externos à organização, atuando como um facilitador na gestão de organizações públicas, privadas e sem fins lucrativos (SAXTON; BENSON, 2005). 
O estudo do CSO no contexto do Terceiro Setor pode ser observado por meio de quatro dimensões: estrutural, cognitiva, relacional e mobilizadora (TONDOLO; BITENCOURT, 2012). A dimensão estrutural se refere à configuração e padrão das ligações entre os atores, sendo as conexões o principal aspecto a ser analisado. Essa dimensão ressalta os laços sociais (NAHAPIET, 2008; NAHAPIET; GHOSHAL, 1998). A dimensão cognitiva constitui-se das representações, interpretações e sistemas de significado compartilhados entre os atores (NAHAPIET, 2008). Assim, a visão compartilhada e o conjunto comum de valores ajudam a desenvolver essa dimensão, uma vez que facilitam as ações individuais e em grupos que beneficiam toda a organização (TSAI; GHOSHAL, 1998).

A dimensão relacional se preocupa com os relacionamentos entre os atores, descrevendo aspectos como vínculos de amizade, normas compartilhadas, obrigações mútuas e identificação, as quais influenciam o comportamento do indivíduo nos contextos sociais (COLEMAN, 1988; NAHAPIET, 2008). Considerando o contexto de pesquisa, esta dimensão é analisada por meio da confiança, sendo uma das principais propulsoras do CSO, e das normas e regras de conduta, sendo essas consideradas como aspectos facilitadores do CSO.

A dimensão mobilizadora refere-se à capacidade da organização social em mobilizar e compartilhar recursos. Essa dimensão é vista como uma forma alternativa de perceber o CSO, tendo sido desenvolvida especialmente para o Terceiro Setor, considerando o caráter estratégico e os aspectos ligados à sustentabilidade dessas organizações (TONDOLO; BITENCOURT, 2012).

Considerados os elementos expostos anteriormente, a Figura 1 apresenta o framework de CSO adotado nesta pesquisa.

Figura 1: Framework de CSO para o Terceiro Setor

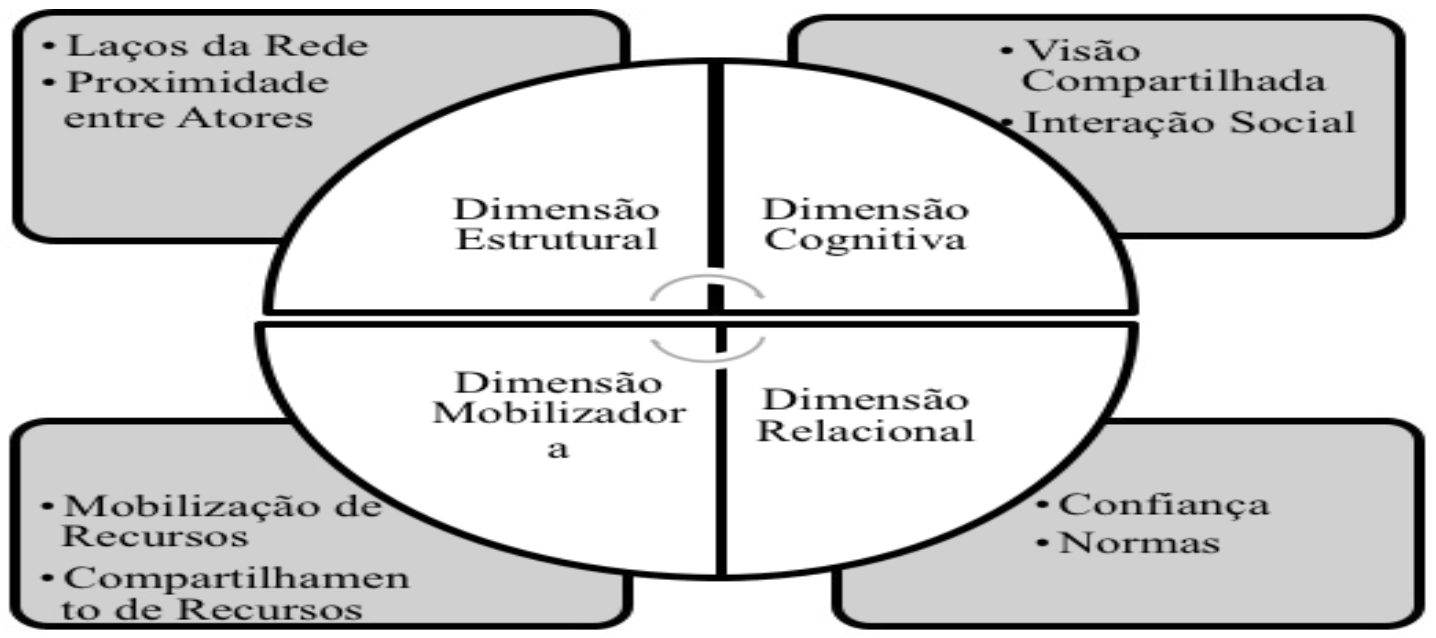

Fonte: Elaborado pelos autores.

\section{ASPECTOS METODOLÓGICOS}

Este estudo tem como objetivo identificar o nível de CSO das organizações sociais participantes de um projeto interorganizacional desenvolvido no Rio Grande do Sul. Para isso, foi realizada uma survey com uma amostra de 44 organizações, sendo que a população envolvia 76 organizações. De acordo com Hair et al. (2005a, 2005b), geralmente, em surveys, o volume de respondentes é considerado baixo se comparado com o número de variáveis. No entanto, esta pesquisa apresentou um percentual de retorno bastante elevado, uma vez que obteve a participação de $57,89 \%$ da população. O nível de significância de corte utilizado para os testes foi de 0,10. 
O instrumento de coleta de dados continha dois blocos de questões. O primeiro era relacionado à 17 variáveis e medindo as 4 dimensões do CSO: estrutural, cognitiva, relacional e mobilizadora, propostos por Tondolo e Bitencourt (2012). A dimensão estrutural apresentou 5 variáveis, dentre as quais 2 buscaram medir os laços da rede e 3 buscaram medir a proximidade entre os atores. A dimensão cognitiva apresentou 4 variáveis, dentre as quais 2 buscaram medir a visão compartilhada e 2 buscaram mensurar a interação social. A dimensão relacional apresentou 4 variáveis, dentre as quais 2 buscaram medir a categoria confiança, enquanto as outras 2 variáveis buscaram medir a categoria normas. E, a dimensão mobilizadora apresentou 4 variáveis, dentre as quais 1 buscou mensurar o compartilhamento de recursos, enquanto 3 buscaram aferir a mobilização de recursos.

As 17 variáveis observadas foram medidas na forma de afirmativas, assim, os respondentes afirmavam seu grau de concordância com cada uma delas. O processo de coleta dos dados utilizou a escala do tipo Likert, de 5 pontos ( 1 = discordo totalmente; 5 = concordo totalmente). Além disso, a confiabilidade da escala, avaliada pelo alfa de Cronbach, foi de 0,756 , considerado adequado para a realização de análises com consistência interna, de acordo com Hair et al. (2005a).

Já o segundo bloco apresentava questões sobre o perfil e informações gerais da organização e do respondente.

Para a realização da análise dos dados quantitativos, os mesmos foram tabulados e analisados com o auxílio do software IBM SPSS, versão 21.0. A análise realizada teve como objetivo inicial identificar os níveis de CSO de cada uma das organizações. Dessa forma, gerou-se uma variável com a média de capital social organizacional, a qual foi baseada nas 17 variáveis que compuseram o construto.

Dispondo dessa informação, foi realizada uma análise de conglomerados (Cluster Analysis), cujo objetivo era classificar as Organizações Sociais, participantes do projeto, quanto as suas médias de Capital Social Organizacional (CSO). O propósito da análise de conglomerados é reunir objetos, classificados de acordo com suas similaridades, com os demais pertencentes àquele grupo (CORRAR; PAULO; DIAS, 2007). Espera-se que cada grupo resultante desta classificação apresente alto grau de homogeneidade interna e alta heterogeneidade externa (CORRAR; PAULO; DIAS, 2007). Nesse sentido, as organizações participantes desta pesquisa foram agrupadas por níveis similares de CSO. Para medir a semelhança entre os objetos, utilizou-se a medida de distância euclidiana quadrada entre pontos.

Com base nos resultados da análise de conglomerados, os testes de Kruskal-Wallis e Wilcoxon-Mann-Whitney foram realizados para confirmar a existência de 3 grupos distintos, se as organizações realmente pertenciam a cada um desses grupos, e que existia diferença entre cada grupo. Nesse sentido, o teste não paramétrico de Kruskal-Wallis, o qual equivale à análise de variância, foi utilizado com o objetivo de identificar se a mediana da variável CSO apresentava diferença entre os grupos.

O teste de Kruskal-Wallis é utilizado para avaliar a similaridade de postos de 2 ou mais grupos independentes que não adiram à populações normais (HO, 2006). "O teste de Kruskal -Wallis é um teste extremamente útil para decidir se $k$ amostras independentes provêm de populações diferentes" (SIEGEL; CASTELLAN, 2006, p. 235), testando a hipótese nula de que k amostras (grupos) provêm da mesma população ou populações idênticas com a mesma mediana (SIEGEL; CASTELLAN, 2006). No entanto, este teste não informa quais os grupos são diferentes, tampouco quantos grupos são diferentes (SIEGEL; CASTELLAN, 2006).

A fim de identificar se os grupos 1, 2 e 3 apresentavam diferenças entre si, foi realizado o teste não paramétrico de Wilcoxon-Mann-Whitney, que equivale ao teste paramétrico T. “O teste 
de Wilcoxon-Mann-Whitney pode ser usado para testar se dois grupos independentes foram extraídos de uma mesma população" (SIEGEL; CASTELLAN, 2006, p. 153). A hipótese nula testada por Wilcoxon-Mann-Whitney é a de que as medianas dos 2 grupos são iguais (GREEN; SALKIND; AKEY, 2000; HO, 2006). Este teste foi aplicado quando detectada a diferença entre grupos, com o objetivo de confirmar se os 3 grupos encontrados na análise de conglomerados eram diferentes a esse nível de significância.

\section{ANÁLISE DOS DADOS}

O projeto interorganizacional, objeto de análise desta pesquisa, foi constituído com o objetivo de capacitar as organizações sociais em princípios de transparência e prestação de contas. Este projeto é fruto de uma parceria entre a organização desenvolvedora do projeto, dois financiadores e uma rede de 21 organizações e instituições, de diversos setores, que constituíram uma rede colaborativa para o desenvolvimento do projeto. Além disso, o projeto investigado contou com a participação de 76 Organizações Sociais no Estado do Rio Grande do Sul, as quais foram capacitadas para a prestação de contas e transparência.

Este projeto apresentou um caráter inovador, uma vez que se trata de um projeto piloto realizado no Brasil, no período de 2009-2011. O objetivo inicial desta patrocinadora foi profissionalizar e aumentar a credibilidade e a visibilidade do Terceiro Setor. Considerando a amplitude do projeto analisado, esta pesquisa delimitou-se a investigar o capital social organizacional das organizações sociais participantes do projeto.

Com relação à amostra analisada, as 44 organizações participantes da pesquisa são oriundas de 17 municípios do Estado do Rio Grande do Sul, e atendem, em média, 1.698 beneficiados. No entanto, vale ressaltar que algumas organizações não atuam com número de beneficiados diretos, pois o foco de atuação destas organizações é a capacitação, reduzindo a média de beneficiados. Foi possível identificar a área de atuação dessas organizações, sendo, na sua maioria, assistência social, educação e saúde. A relação de organizações sociais respondentes, por município, pode ser observada na Tabela 1.

Tabela 1: Relação das organizações sociais por município

\begin{tabular}{c|c}
\hline Cidades & Quantidade de Organizações \\
\hline Cachoeirinha & 1 \\
\hline Canoas & 1 \\
\hline Charqueadas & 2 \\
\hline Erechim & 1 \\
\hline Faxinal do Soturno & 1 \\
\hline Frederico Westphalen & 1 \\
\hline Giruá & 1 \\
\hline Novo Hamburgo & 1 \\
\hline Porto Alegre & 19 \\
\hline Rio Grande & 1 \\
\hline Rio Pardo & 1 \\
\hline Santa Maria & 8 \\
\hline Santa Rosa & 1 \\
\hline Santo Antônio da Patrulha & 1 \\
\hline Sapucaia do Sul & 2 \\
\hline Uruguaiana & 1 \\
\hline Venâncio Aires & 1 \\
\hline
\end{tabular}

Fonte: Elaborado pela autora. 


\subsection{Análise de Conglomerados}

Após a análise introdutória dos dados e buscando atingir o objetivo geral desta pesquisa, optou-se por analisar os dados quantitativos a partir da técnica de análise de conglomerados, uma vez que a pesquisa objetivou identificar e classificar os níveis de CSO declarado pelas organizações sociais participantes do projeto. Para isso, gerou-se a média das 17 variáveis, a fim de identificar a média de CSO de cada uma das organizações, o que pode ser visto na Figura 2. Pode-se observar que, conforme a escala utilizada, o CSO médio declarado pelos respondentes distribuiu-se entre 3,29 e 5,00 pontos.

Figura 2: Média de Capital Social Organizacional

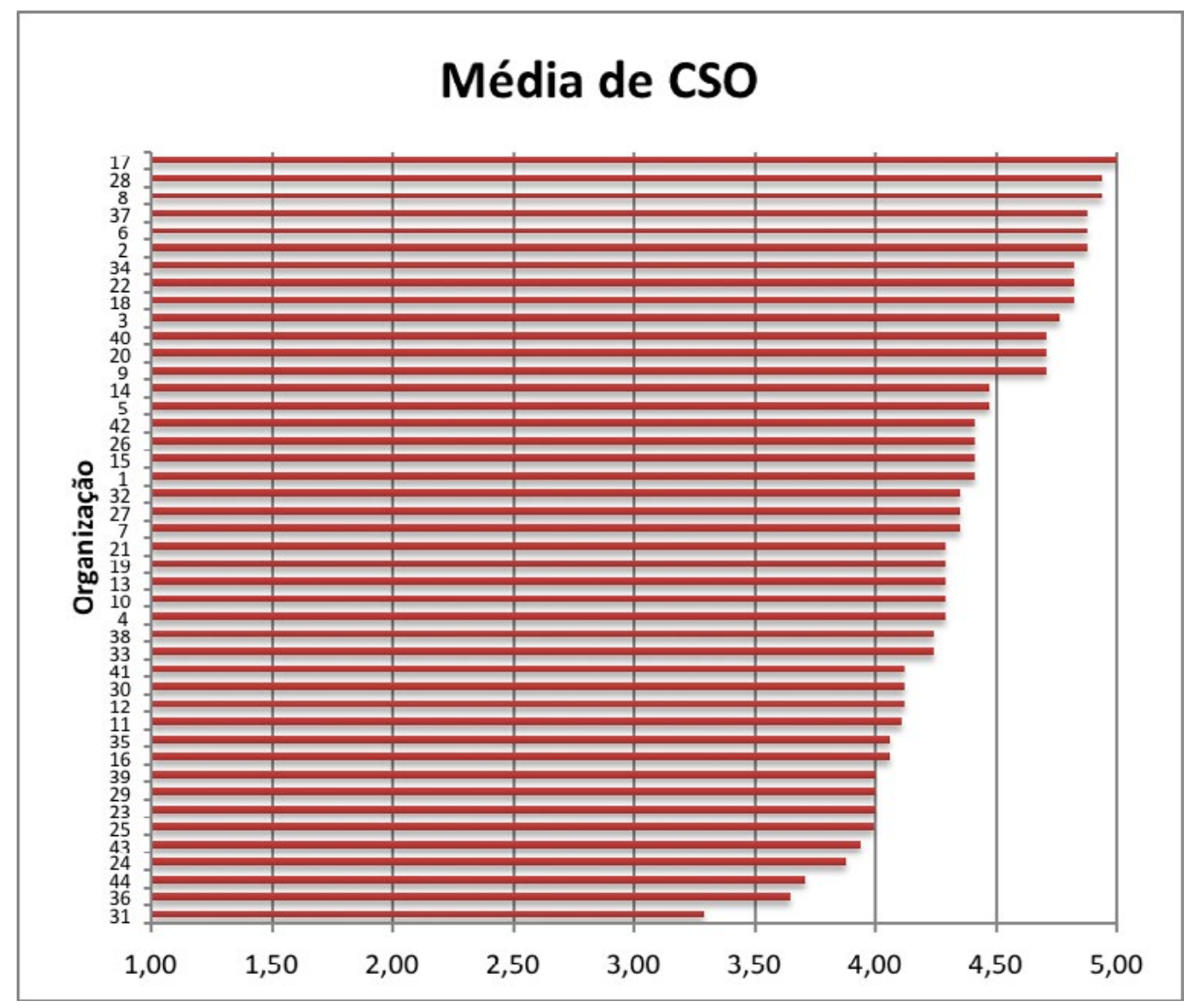

Fonte: Elaborado pelos autores.

Posteriormente, verificou-se a semelhança a partir da distância euclidiana quadrada entre os pontos, utilizando-se da análise de cluster hierárquica. Essa técnica é empregada de forma exploratória, por ser robusta à existência de outliers e não demandar normalidade dos dados (RENCHER, 2002). A partir desta análise, foram identificados os membros de cada um dos conglomerados, gerando 3 agrupamentos. A partir da identificação das organizações pertencentes a cada um dos 3 conglomerados, foi possível classificá-las da seguinte forma:

a. Níveis elevados de CSO - foram identificadas 13 organizações, as quais apresentaram escore médio de CSO de 4,84, e, com $90 \%$ de confiança, entre 4,78 e 4,90. Este agrupamento foi denominado $\mathrm{C} 1$;

b. Níveis medianos de CSO - foram identificadas 28 organizações, as quais apresenta- 
ram escore médio de CSO de 4,21, e, com $90 \%$ de confiança, entre 4,15 e 4,28. Este agrupamento foi denominado $\mathrm{C} 2$;

c. Níveis baixos de CSO - foram identificadas 3 organizações, as quais apresentaram escore médio de CSO de 3,55, e, com $90 \%$ de confiança, entre 3,00 e 4,10. Este agrupamento foi denominado $\mathrm{C} 3$.

A partir dessa análise, foi possível concluir que dentre os respondentes existiam 3 grupos distintos quanto aos níveis de capital social organizacional, que se diferenciaram por seus escores médios de CSO. A fim de analisar a constituição dos grupos e as diferenças entre eles, foram realizados os testes de Kruskal-Wallis, Mann-Whitney $U$ e Wilcoxon, que serão apresentados na discussão dos dados.

\subsection{Análise dos Grupos}

O Teste de Kruskal-Wallis usou a hipótese nula $\left(\mathrm{H}_{0}\right)$ de que as medianas da distribuição de CSO médio são iguais entre os três grupos. Diante disso, sendo a hipótese alternativa verdadeira, ao menos um grupo deve apresentar medianas diferentes (SIEGEL; CASTELLAN, 2006). Nesse sentido, apresenta-se a classificação a partir dos grupos analisados, os integrantes desses grupos e as médias da variável CSO para cada um dos grupos. Assim, o conglomerado C1 apresenta 13 integrantes e classificação média de CSO igual a 38,00. O conglomerado C2 apresenta 28 integrantes e classificação média de CSO igual a 17,50. Já o conglomerado C3 apresenta 3 integrantes e classificação média de CSO igual a 2,00. Estas informações podem ser vistas na Tabela 1.

Tabela 1: Classificação das médias de CSO

\begin{tabular}{c|c|c}
\hline Clusters & N & Média de CSO \\
\hline C1 & 13 & 38,00 \\
\hline C2 & 28 & 17,50 \\
\hline C3 & 3 & 2,00 \\
\hline Total & 44 & \\
\hline
\end{tabular}

Fonte: Elaborado pelos autores.

Considerando 2 (3-1) graus de liberdade e a estatística qui-quadrado obtida, de 30,94, obtém-se uma significância assintótica $p<0,001$. Desta forma, o teste rejeita $\mathrm{H}_{0}$ e evidencia diferenças significantes entre os grupos. Para os clusters estabelecidos, foram também analisadas, com base no teste de Kruskal-Wallis, as diferenças dos valores médios de cada uma das variáveis que compõem as dimensões em estudo, relacionadas ao CSO. Os níveis de significância e o quiquadrado de cada uma das variáveis são apresentados na Tabela 2. 
Tabela 2: Níveis de significância de cada variável

\begin{tabular}{|c|c|c|c|c|c|c|}
\hline \multirow{4}{*}{$\begin{array}{l}\text { Dimensão } \\
\text { Estrutural }\end{array}$} & Variáveis & $\begin{array}{l}\text { Laços da } \\
\text { Rede } 1\end{array}$ & $\begin{array}{c}\text { Laços } \\
\text { da Rede } \\
2\end{array}$ & $\begin{array}{l}\text { Proximidade } \\
\text { entre atores } 1\end{array}$ & $\begin{array}{l}\text { Proximidade } \\
\text { entre atores } 2\end{array}$ & $\begin{array}{l}\text { Proximidade } \\
\text { entre atores } 3\end{array}$ \\
\hline & Qui-Quadrado & 8,177 & 6,926 & 12,508 & 16,105 & 16,806 \\
\hline & $\begin{array}{c}\text { Graus de li- } \\
\text { berdade }\end{array}$ & 2 & 2 & 2 & 2 & 2 \\
\hline & $\begin{array}{l}\text { Significância } \\
\text { Assintótica }\end{array}$ & 0,017 & 0,031 & 0,002 & 0,000 & 0,000 \\
\hline \multirow{4}{*}{$\begin{array}{l}\text { Dimensão } \\
\text { Cognitiva }\end{array}$} & Variáveis & \begin{tabular}{|c|} 
Visão \\
Compar- \\
tilhada 1
\end{tabular} & \begin{tabular}{|c|} 
Visão \\
Com- \\
parti- \\
lhada 2
\end{tabular} & $\begin{array}{l}\text { Interação So- } \\
\text { cial } 1\end{array}$ & $\begin{array}{l}\text { Interação So- } \\
\text { cial } 2\end{array}$ & \\
\hline & Qui-Quadrado & 6,200 & 3,221 & 8,127 & 18,355 & \\
\hline & $\begin{array}{c}\text { Graus de li- } \\
\text { berdade }\end{array}$ & 2 & 2 & 2 & 2 & \\
\hline & $\begin{array}{l}\text { Significância } \\
\text { Assintótica }\end{array}$ & 0,045 & 0,200 & 0,017 & 0,000 & \\
\hline \multirow{4}{*}{$\begin{array}{l}\text { Dimensão } \\
\text { Relacional }\end{array}$} & Variáveis & \begin{tabular}{|c|}
$\begin{array}{c}\text { Confian- } \\
\text { ça } 1\end{array}$ \\
\end{tabular} & $\begin{array}{c}\text { Con- } \\
\text { fiança } 2 \\
\end{array}$ & Normas 1 & Normas 2 & \\
\hline & Qui-Quadrado & 12,068 & 4,589 & 5,857 & 17,913 & \\
\hline & $\begin{array}{c}\text { Graus de li- } \\
\text { berdade }\end{array}$ & 2 & 2 & 2 & 2 & \\
\hline & $\begin{array}{c}\text { Significância } \\
\text { Assintótica } \\
\end{array}$ & 0,002 & 0,101 & 0,053 & 0,000 & \\
\hline \multirow{4}{*}{$\begin{array}{c}\text { Dimensão } \\
\text { Mobiliza- } \\
\text { dora }\end{array}$} & Variáveis & \begin{tabular}{|c|} 
Com- \\
partilha- \\
mento \\
de Re- \\
cursos 1
\end{tabular} & $\begin{array}{c}\text { Mobi- } \\
\text { lização } \\
\text { de Re- } \\
\text { cursos } 1\end{array}$ & $\begin{array}{l}\text { Mobilização } \\
\text { de Recursos } 2\end{array}$ & $\begin{array}{c}\text { Mobilização } \\
\text { de Recursos } 3\end{array}$ & \\
\hline & Qui-Quadrado & 7,814 & 7,460 & 6,300 & 11,559 & \\
\hline & $\begin{array}{c}\text { Graus de li- } \\
\text { berdade }\end{array}$ & 2 & 2 & 2 & 2 & \\
\hline & $\begin{array}{l}\text { Significância } \\
\text { Assintótica }\end{array}$ & 0,020 & 0,024 & 0,043 & 0,003 & \\
\hline
\end{tabular}

Fonte: Elaborado pelos autores.

Desta forma, das 17 variáveis utilizadas por este estudo, apenas 2 não apresentaram diferenças significantes entre os agrupamentos identificados. Como o teste de Kruskal Wallis apresenta como limitador a indicação de que somente um grupo é diferente de algum dos demais, esta pesquisa buscou complementar as análises a partir do teste de Mann-Whitney e Wilcoxon, descrito a seguir.

\subsection{Teste de Mann-Whitney e Wilcoxon}

Para a realização do Teste de Mann-Whitney e Wilcoxon, foi necessária a classificação entre 2 grupos. Os primeiros grupos classificados foram os grupos 1 e 2, e o critério utilizado foi a variável média de CSO. Desta forma, obteve-se a classificação por média de cada grupo, nesta variável, e por somatório (R). Assim, obtiveram-se 41 organizações no total, sendo 13 do cluster C1 e 28 oriundas do cluster C2. O cluster $\mathrm{C} 1$ apresentou classificação média de 35,00 e somatório de 455,00, enquanto o cluster C2 apresentou classificação média de 14,50 e somatório de 406,00.

O teste estatístico de Mann-Whitney (U) apresentou o valor 0,000, já o Wilcoxon (W) de 406,000. Estes testes apresentaram um nível de significância de 0,000; assim, são considerados 
diferentes, ou seja, a variável média CSO é diferente nos cluster C1 e C2. Desta forma, pode-se afirmar que os níveis de CSO em cada um desses 2 grupos são diferentes, ou seja, o nível de CSO do grupo C1 é maior do que do grupo C2. Esses valores podem ser acompanhados na Tabela 3.

Tabela 3: Teste estatístico de Mann-Whitney e Wilcoxon - Grupo 1 e 2

\begin{tabular}{c|c}
\hline & Média CSO \\
\hline Mann-Whitney U &, 000 \\
\hline Wilcoxon W & 406,000 \\
\hline Z & $-5,112$ \\
\hline Asymp. Sig. (2-tailed) &, 000 \\
\hline Exact Sig. [2*(1-tailed Sig.)] &, $000(\mathrm{a})$ \\
\hline
\end{tabular}

Fonte: Elaborado pelos autores.

Quando comparados os clusters C2 e C3, obtiveram-se 31 organizações no total, sendo 28 oriundas do cluster $\mathrm{C} 2$ e 3 do cluster $\mathrm{C} 3$. O cluster $\mathrm{C} 2$ apresentou classificação média de 17,50 e somatório de 490,00, enquanto o cluster C3 apresentou classificação média de 2,00 e somatório de 6,00.

Já o teste estatístico de Mann-Whitney (U), para estes grupos, apresentou o valor de 0,000 , e o Wilcoxon (W) de 6,000. Estes testes apresentaram um nível de significância de 0,005; assim, são considerados diferentes, ou seja, a variável média CSO é diferente nos cluster C2 e C3. Desta forma, pode-se afirmar que os níveis de CSO em cada um desses 2 grupos são diferentes. Então, o grupo C2 apresenta nível de CSO superior ao grupo C3. Esses valores podem ser acompanhados na Tabela 4.

Tabela 4: Teste estatístico de Mann-Whitney e Wilcoxon - Grupos C2 e C3

\begin{tabular}{c|c}
\hline & Média CSO \\
\hline Mann-Whitney U &, 000 \\
\hline Wilcoxon W & 6,000 \\
\hline Z & $-2,819$ \\
\hline Asymp. Sig. (2-tailed) &, 005 \\
\hline Exact Sig. [2*(1-tailed Sig.)] &, $000(\mathrm{a})$ \\
\hline
\end{tabular}

Fonte: Elaborado pelos autores.

Quando analisado os clusters C1 e C3, obtiveram-se 16 organizações no total, sendo 13 oriundas do cluster $\mathrm{C} 1$ e 3 do cluster $\mathrm{C} 3$. O cluster $\mathrm{C} 1$ apresentou classificação média de 10,00 e somatório de 130,00, enquanto o cluster C3 apresentou classificação média de 2,00 e somatório de 6,00.

Nesta análise, o teste estatístico de Mann-Whitney $(U)$ apresentou o valor de 0,000 , já o Wilcoxon (W) de 6,000. Estes testes apresentaram um nível de significância exata ${ }^{1}$ de 0,004; assim, são considerados diferentes, ou seja, a variável média CSO é diferente nos cluster C1 e C3. Desta forma, pode-se afirmar que os níveis de CSO em cada um desses 2 grupos são diferentes. Então, o grupo C1 apresenta nível de CSO superior ao grupo C3. Esses valores podem ser acompanhados na Tabela 5.

Tabela 5: Teste estatístico de Mann-Whitney e Wilcoxon - Grupo 2 e 3

\begin{tabular}{c|c}
\hline & Média CSO \\
\hline Mann-Whitney U &, 000 \\
\hline Wilcoxon W & 6,000 \\
\hline Z & $-2,649$ \\
\hline Asymp. Sig. (2-tailed) &, 008 \\
\hline Exact Sig. [2*(1-tailed Sig.)] &, $004(\mathrm{a})$
\end{tabular}

Fonte: Elaborado pelos autores.

1 Foi utilizada a significância exata porque $\mathrm{N}<30$ (MARÔCO, 2011). 
Assim, a partir dos testes estatísticos realizados, conclui-se que os clusters 1, 2 e 3 são estatisticamente diferentes. Dessa forma, esta análise corrobora a afirmação de que a amostra de 44 organizações é formada por 3 grupos com níveis distintos de CSO.

\subsection{Discussão dos Resultados}

Este estudo buscou mensurar o nível de CSO de 44 organizações participantes de um projeto interorganizacional realizado no Terceiro Setor Gaúcho. Para isso, foi utilizado um instrumento contendo 17 variáveis, que compõem as 4 dimensões (estrutural, cognitiva, relacional e mobilizadora).

A dimensão estrutural foi constituída por 5 variáveis, aglutinadas em 2 categorias (Laços da Rede e Proximidade entre Atores), as quais buscaram medir os laços estabelecidos entre as organizações, bem como a proximidade das organizações sociais com as demais organizações participantes do projeto. Desta forma, os testes estatísticos comprovaram que as 5 variáveis utilizadas para mensurar a dimensão estrutural do CSO são significantes, pois validam e efetivam a mensuração de CSO estrutural no projeto realizado no Terceiro Setor. Por se tratar de um projeto interorganizacional, a dimensão estrutural apresenta grande relevância, uma vez que existem muitos atores envolvidos e o bom desempenho no CSO estrutural pode influenciar nos resultados organizacionais obtidos.

Já a dimensão cognitiva apresentou 4 variáveis, agrupadas em 2 categorias (Visão Compartilhada e Interação Social), as quais buscaram medir o compartilhamento de visões e ambições entre as organizações, a motivação da equipe da organização social, e a interação social, investigada a partir do relacionamento com outras organizações participantes do projeto, bem como pela reação a um convite destinado a socialização com as organizações participantes. Os testes estatísticos apresentaram a significância de 3 das 4 variáveis propostas. A variável Visão Compartilhada 2, que apresentou a pergunta "As pessoas na sua OSC estão coletivamente entusiasmadas com a participação no projeto transparência?", apresentou nível de significância assintótica de 0,200, de tal modo, esta questão não é significativa para os respondentes, uma vez que não apresenta a compreensão adequada. Assim, a permanência desta variável no instrumento de pesquisa deve ser revista.

A dimensão cognitiva do CSO é onde a organização desenvolve a construção de sentido perante outras organizações, ou seja, é a partir da identificação que a organização consegue desenvolver capacidades, como a visão compartilhada e a interação social. Essas capacidades são aprimoradas por meio das relações, gerando resultados organizacionais. Em um projeto interorganizacional, essa dimensão do CSO tende a se intensificar, uma vez que existem muitas organizações de diferentes patamares e origens, envolvidas nos processos, e a busca pela identificação com as demais é ativada por meio das interações.

A dimensão relacional apresentou 4 variáveis, as quais buscaram mensurar a confiança nos demais participantes do projeto e o estabelecimento de regras e normas. Os testes estatísticos apresentaram que a variável Confiança 2 apresentou nível de significância de 0,101, assim estando fora do nível de significância. No entanto, por estar muito próxima ao limite, que é 0,10 , esta variável indica uma tendência a ser observada, ainda que, pelo critério estabelecido, não apresente diferença significante entre os agrupamentos identificados. As demais variáveis foram significantes e retratam a dimensão relacional do CSO. Esta dimensão, no contexto de um projeto interorganizacional, é relevante porque apresenta o padrão das relações estabelecidas, uma vez que demonstra a confiança entre os atores, bem como as regras de conduta estabelecidas para a ocorrência dessas relações.

Por fim, a dimensão mobilizadora apresentou 4 variáveis dispostas em 2 categorias (mobilização de recursos e compartilhamento de recursos). Os testes estatísticos apresentaram a significância das 4 variáveis analisadas, ou seja, para os respondentes, as questões propostas foram 
significantes e faziam sentido. Desta forma, é possível afirmar que o CSO Mobilizador é expresso por meio das 2 categorias citadas anteriormente, as quais buscam mensurar se a organização social é capaz de identificar o recurso e a capacidade necessária, articulá-los, captá-los e implementá-los nas ações sociais, bem como, se as organizações conseguem compartilhar os recursos/ capacidades entre os participantes e explorá-las de forma efetiva.

A dimensão mobilizadora contribui para o contexto de um projeto interorganizacional na medida em que identifica a possibilidade de contribuir, de forma efetiva, para a compreensão dos aspectos relacionados à mobilização de recursos, realizada pelas organizações do Terceiro Setor. Nesta dimensão, o CSO é retratado como uma capacidade que as organizações precisam identificar e explorar de forma efetiva, para contribuir com os processos e o desempenho organizacional.

Refletindo sob a ótica dos Projetos Interorganizacionais desenvolvidos no Terceiro Setor, o CSO apresenta-se como aspecto chave, uma vez que o envolvimento das organizações no desenvolvimento dos projetos é crucial, e os laços e relações estabelecidas contribuirão de forma efetiva para os resultados, tanto individuais, do projeto, quanto para os resultados coletivos, que envolverão as organizações participantes. Além disso, vale ressaltar que são esses ambientes que contribuem para a realização de uma intersecção entre os três setores, ou seja, os projetos interorganizacionais são ambientes propícios para ampliar as redes e fortalecer os vínculos de relações entre as organizações, assim aprimorando e desenvolvendo capacidades, visando à mobilização de recursos.

Diante disso, a presença de 3 grupos com distintos níveis de CSO destaca o potencial das organizações sociais em se articular e mobilizar recursos e capacidades. Por outro lado, ressalta a heterogeneidade dessas organizações, uma vez que, imersas em um mesmo ambiente, obtiveram níveis de CSO diferenciados. Esse aspecto enfatiza o que os resultados estatísticos comprovaram: que as organizações são semelhantes intragrupos, mas diferentes entre grupos.

No entanto, deve ser considerado que essas organizações apresentam características fortes por estarem associadas a públicos e propósitos diferenciados, uma vez que essas organizações são regidas, na sua maioria, por um estímulo muitas vezes inexplicável, onde aspectos motivacionais movem grupos de pessoas a agirem em prol de uma causa específica (BITENCOURT et al., 2007). Essa comoção envolvendo as pessoas pode ser vista como um motivador na busca pela melhoria dos processos e desenvolvimento de novas capacidades.

\section{CONCLUSÕES}

Este estudo identificou, por meio de uma pesquisa quantitativa, o nível de capital social organizacional das organizações sociais participantes de um projeto interorganizacional, desenvolvido no Estado do Rio Grande do Sul. Diante disso, foi possível identificar 3 grupos com níveis diferentes de CSO declarado. Estes grupos foram chamados de níveis baixo, mediano e elevados de CSO.

Além disso, este estudo contribui para a validação do instrumento proposto por Tondolo e Bitencourt (2012), uma vez que o construto sugerido pelas autoras foi utilizado para realização desta pesquisa, e obteve um alfa de Cronbach de 0,756, considerado adequado, de acordo com Hair et al (2005a).

Dessa forma, entende-se a Dimensão Mobilizadora como uma capacidade organizacional das organizações sociais, a partir de seus elementos constitutivos, mobilização e compartilhamento de recursos. Essa capacidade é desenvolvida durante a trajetória organizacional e apresenta-se fortemente vinculada às peculiaridades das organizações que integram este setor. É preciso destacar a contribuição da dimensão mobilizadora para os avanços teóricos, envolvendo tanto a teoria do capital social quanto as abordagens referentes ao Terceiro Setor. Esta dimensão tem como base a capacidade de mobilizar os recursos tangíveis e intangíveis, uma vez que a mobilização é entendida, 
neste estudo, pela ação de identificar, buscar e implementar os recursos e capacidades necessários pela organização para atingimento de seus objetivos sociais (SIRMON; HITT; IRELAND, 2007).

A visão do CSO, no contexto do terceiro setor, apresenta-se como um recurso/capacidade, uma vez que o seu desenvolvimento pode influenciar de diferentes formas as organizações sociais, seja como um facilitador na gestão, a partir de fatores internos e externos (SAXTON; BENSON, 2005), ou ainda, nas diferenças de desempenho oriundas da habilidade para criar e explorar o capital social (NAHAPIET; GHOSHAL, 1998). De acordo com Pennings e Lee (1998), o capital social é exclusivo e difícil de apropriar, porque depende do envolvimento contínuo de duas ou mais partes. Nesse sentido, o projeto interorganizacional surge como um ambiente propício ao desenvolvimento de CSO, na medida em que mobiliza diversos atores em prol de um objetivo comum. No entanto, vale ressaltar o desafio para gerenciar as relações estabelecidas entre os atores, bem como o estímulo para essas relações e manutenção do foco, e objetivo comum.

Este estudo apresenta potenciais implicações gerenciais, tais como: (i) o CSO pode ser visto como um diferencial na gestão de projetos interorganizacionais, uma vez que níveis relativamente elevados de CSO podem vir a contribuir para um melhor desempenho do projeto e das organizações participantes. Os níveis elevados de CSO podem ser alcançados por meio das interações entre as organizações participantes, sendo estas relações alicerçadas em confiança, e promovem a mobilização e o compartilhamento de recursos; (ii) a busca por recursos depende do CSO, uma vez que é a partir das relações estabelecidas entre os atores que os recursos são articulados e mobilizados. Dessa forma, o CSO é uma capacidade que as organizações sociais precisam desenvolver e que está diretamente associada ao desempenho da organização; (iii) o CSO deve ser visto como um aspecto central na gestão das organizações sociais, pois contribui de forma efetiva para a mobilização e compartilhamento de recursos. Assim, a sua manutenção e crescimento são importantes para o atingimento da missão organizacional e para a perpetuidade da organização na sociedade.

Como limitação, o estudo centraliza-se na percepção do respondente, uma vez que se mensurou o CSO por meio da sua visão. Nesse sentido, buscou-se minimizar esse viés através da escolha dos respondentes, ou seja, foram selecionados respondentes que fossem membros da gestão da organização social.

Ainda existem muitas questões a serem exploradas sobre o CSO, e uma delas envolve a compreensão da interação entre CSO e o desempenho no nível organizacional e interorganizacional (NAHAPIET, 2008). Diante disso, sugere-se quem em estudos futuros seja investigado o comportamento das variáveis "visão compartilhada 2" e "confiança 2", as quais não apresentaram diferença significante neste estudo.

Já a variável "laços da rede 2" apresentou um comportamento diferente, uma vez que o grupo 1 detém a menor classificação média, enquanto o grupo 3, que deveria apresentar essa característica, apresenta valor intermediário entre os grupos. Vale ressaltar que esta é a única variável de todo construto que apresentou este tipo de comportamento. Essas limitações não foram investigadas em profundidade por este estudo, por isso sugere-se que estudos futuros dediquem atenção à compreensão destes resultados. E, sugere-se ainda, que seja investigada a relação entre os níveis de CSO e a forma como os recursos são mobilizados pelas organizações, verificando se níveis mais elevados de CSO apresentam formas distintas, e mais robustas, de mobilizar seus recursos.

Ao final, vale ressaltar as contribuições deste estudo, as quais envolvem a mensuração e classificação de 3 grupos de organizações sociais com diferentes níveis de CSO. Estando estas organizações presentes no contexto de um projeto interorganizacional, estes resultados contribuem para o contexto empírico e teórico, uma vez que possibilitam, tanto a acadêmicos quanto 
a gestores do Terceiro Setor, a reflexão sobre os motivos e razões da existência de 3 diferentes grupos de CSO, e se estas organizações tinham o mesmo cenário de atuação (projeto interorganizacional), além de refletir sobre as ações a serem desenvolvidas pelas organizações para melhorar os níveis de CSO. Estas reflexões são importantes porque estão diretamente ligadas à arrecadação de recursos tangíveis e intangíveis.

\section{REFERENCIAS}

ADLER; P. S.; KWON, S. Social Capital: Prospects for a New Concept. Academy of Management Review, v. 27, n. 1, p. 17-40, 2002.

ARREGLE, J. et tal. The Development of Organizational Social Capital: attributes of family firms. Journal of Management Studies, v. 44, n. 1, p. 73-95, 2007.

BITENCOURT, C. et al. Doing better by doing good: the experience of a virtual community network in Brazil. In: ACADEMY OF MANAGEMENT, 2007, Philadelphia. Anais... Philadelphia: AOM, 2007.

BOLINO, M. C.; TURNLEY, W. H.; BLOODGOOD, J. M. Citizenship Behavior and the Creation of Social Capital in Organizations. Academy of Management Review, v. 27, n. 4, p. 505-522, 2002.

COLEMAN, J. S. Social capital in the creation of human capital. The American Journal of Sociology, v. 94, p. 95-120, 1988.

CORRAR, L.J.; PAULO, E.; DIAS FILHO, J.M. Análise Multivariada: para os cursos de administração, ciências contábeis e economia. São Paulo: Atlas, 2007.

CRUZ, J. A. W.; QUANDT, C. O.; MARTINS, T. S.; SILVA, W. V. Performance no Terceiro Setor Uma abordagem de accountability. Revista de Administração da UFSM, v. 3, n. 1, p. 58-75, 2010.

EDELMAN, L. F. et tal. The Benefits and Pitfalls of Social Capital: Empirical evidence from two organizations in the United Kingdom. British Journal of Management, v. 15, p. 59-69, 2004.
GREEN, S. B.; SALKIND, N. J.; AKEY, T. M. Using SPSS for windows: analyzing and understanding data. 2. ed. Upper Saddle River: Prentice-hall, 2000.

HAIR JÚNIOR, J. et al. Fundamentos de métodos de pesquisa em administração. Porto Alegre: Bookman, 2005a.

Análise Multivariada de Dados. 5. ed. Porto Alegre: Bookman, 2005b.

HO, R. Handbook of univariate and multivariate data analysis and interpretation with SPSS. Boca Raton: Chapman \& Hall/CRC, 2006.

LEANA, C. R.; VAN BUREN, H. J. Organizational Social Capital and Employment Practices. Academy of Management Review, v. 24, n. 3, p. 538-555, 1999.

MARÔCO, J. Análise Estatística com o SPSS Statistics. 5. ed. Pero Pinheiro: Report Number, 2011.

NAHAPIET, J. The Role of Social Capital in Interorganizational Relationships. In: CROPPER, $S$. et tal. The Oxford handbook of interorganizational relations. Oxford: Oxford University Press, 2008. p. 580-606.

NAHAPIET, J.; GHOSHAL, S. Social capital, intellectual capital and the organizational advantage. Academy of Management Review, v. 23, n. 2, p. 242-266, 1998.

OLIVEIRA, L. Capacidades diferenciadoras como vantagem competitiva nas empresas torrefadoras de café. Revista de Administração da UFSM, v.1, n.1, p. 85-100, 2008. 
ORO, I. M.; BALBINOT, I. J.; THOMÉ, D.; LAVARDA, C. E. F. Relação do Capital Intelectual de Natureza Social e Ambiental com o Desempenho Econômico-Financeiro do Segmento de Energia Elétrica Brasileiro. Revista de Administração da UFSM, v. 7, Edição Especial, p. 59-74, 2014.

PENNINGS, J. M.; LEE, K. Social Capital of Organization: Conceptualization, level of analysis and performance implications. In: GABBAY, S.; LEENDERS, R. (Edit.). Corporate Social Capital. 4. ed. New York: Addison Wesley, 1998.

RENCHER, A. C. Methods of Multivariate Analysis. 2nd ed. John Wiley \& Sons. Canada, 2002.

SALAMON, L. M. The Rise of the Nonprofit Sector. Foreign Affairs, v. 73, n. 4, p. 109-122, 1994.

SALAMON, L. M. Putting the Civil Society Sector on the Economic Map of the World. Annals of Public and Cooperative Economics, v. 81, n. 2, p. 167-210, 2010.

SARAIVA, L. A. S. Além do Senso Comum Sobre o Terceiro Setor: uma provocação. In: PIMENTA, S. M.; SARAIVA, L. A. S.; CORRÊA, M. L. (Org.). Terceiro Setor: Dilemas e Polêmicas. São Paulo: Saraiva, 2006. p. 19-40.

SAXTON, G. D.; BENSON, M. A. Social Capital and the Growth of the Nonprofit Sector. Social Science Quarterly, v. 86, n. 1, p. 16-35, 2005.

SIEGEL, S.; CASTELLAN JÚNIOR, N. J. Estatística não-paramétrica para ciências do comportamento. 2. ed. Porto Alegre: Artmed, 2006.

SIRMON, D. G; HITT, M. A.; IRELAND, R. D. Managing Firm Resources in Dynamic Environments to Creste Value: Looking inside the black box. Academy of Management Review, v. 32, n. 1, p. 273-292, 2007.

TSAI, W.; GHOSHAL, S. Social capital and value creation: the role of intrafirm networks. Academy of Management Journal, v. 41, n. 4, p. 464-476, 1998.

TONDOLO, R. R. P.; BITENCOURT, C. C. Mensuração do Capital Social em Projetos Desenvolvidos no Terceiro Setor: uma proposta a partir do projeto Transparência e Prestação de Contas em OSCs no Rio Grande do Sul. In: ENCONTRO DA ANPAD - EnANPAD, XXXVI, 2012, Rio de Janeiro. Anais... Rio de Janeiro: Anpad, 2012.

ZAHRA, S. A. Harvesting Family Firm's Organizational Social Capital: A Relational Perspective. Journal of Management Studies, v. 47, n. 2, p. 345-366, 2010. 